\title{
ALMOST PARA-HERMITIAN AND ALMOST PARACONTACT METRIC STRUCTURES INDUCED BY NATURAL RIEMANN EXTENSIONS
}

\author{
CORNELIA-LIVIA BEJAN AND GALIA NAKOVA
}

\begin{abstract}
In this paper we consider a manifold $(M, \nabla)$ with a symmetric linear connection $\nabla$ which induces on the cotangent bundle $T^{*} M$ of $M$ a semiRiemannian metric $\bar{g}$ with a neutral signature. The metric $\bar{g}$ is called natural Riemann extension and it is a generalization (made by M. Sekizawa and O. Kowalski) of the Riemann extension, introduced by E. K. Patterson and A. G. Walker (1952). We construct two almost para-Hermitian structures on $\left(T^{*} M, \bar{g}\right)$ which are almost para-Kähler or para-Kähler and prove that the defined almost para-complex structures are harmonic. On certain hypersurfaces of $T^{*} M$ we construct almost paracontact metric structures, induced by the obtained almost paraHermitian structures. We determine the classes of the corresponding almost paracontact metric manifolds according to the classification given by S. Zamkovoy and G. Nakova (2018). We obtain a necessary and sufficient condition the considered manifolds to be paracontact metric, K-paracontact metric or para-Sasakian.
\end{abstract}

\section{INTRODUCTION}

The geometry of an almost para-Hermitian manifold $(N, P, g)$ is determined by the action of the almost para-complex structure $P$ as an anti-isometry with respect to the semi-Riemannian metric $g$ in each tangent fibre. The metric $g$ is necessarily of neutral signature. A classification of the almost para-Hermitian manifolds is made by C.-L. Bejan in [1]. The geometry of the almost paracontact metric manifolds is a natural extension of the geometry of the almost para-Hermitian manifolds to the odd dimensional case. Twelve basic classes of almost paracontact metric manifolds $(M, \varphi, \xi, \eta, g)$ with respect to the covariant derivative of the structure tensor $\varphi$ is obtained by S. Zamkovoy and G. Nakova in [12]. Moreover, in [12] it is shown that 3-dimensional almost paracontact metric manifolds belong only to four basic classes from the classification and examples for each of these classes are constructed.

Let $(M, \nabla)$ be an $n$-dimensional manifold endowed with a symmetric linear connection $\nabla$. Patterson and Walker defined in [6] a semi-Riemannian metric on the cotangent bundle $T^{*} M$ of $(M, \nabla)$, called Riemann extension. This metric is of neutral signature $(n, n)$ and it was generalized by M. Sekizawa and O. Kowalski in [5, 8] to natural Riemann extension $\bar{g}$ which has the same signature. Recently, the metric $\bar{g}$ has been studied from different points of view. For instance, Bejan and Kowalski

Date: 9th November 2018.

Key words and phrases. Cotangent bundle, Natural Riemann extension, Almost para-Hermitian manifold, Almost paracontact metric manifold. 
characterized in [4] some harmonic functions on $\left(T^{*} M, \bar{g}\right)$. In [2] Bejan and Eken defined a canonical almost para-complex structure on $\left(T^{*} M, \bar{g}\right)$ and investigated its harmonicity with respect to $\bar{g}$. In 3 the authors constructed a family of hypersurfaces of $\left(T^{*} M, \bar{g}\right)$ which are Einstein manifolds with a positive scalar curvature.

Our aim in the present work is to obtain new examples of almost para-Hermitian and almost paracontact metric manifolds. The paper is organized as follows. In Sect. 2 we recall some notions and results about the cotangent bundle of a manifold and the lifting of objects from the base manifold to its cotangent bundle. In Sect. 3, motivated from the fact that the natural Riemann extension $\bar{g}$ on $T^{*} M$ is of neutral signature, we construct two almost para-Hermitian structures $(P, \bar{g})$ and $\left(P_{1}, \bar{g}_{1}\right)$ on $T^{*} M$, where $\bar{g}$ and $\bar{g}_{1}$ are proper and non-proper natural Riemann extension, respectively. We prove that in the case when $M$ is not flat (resp. $M$ is flat) both manifolds $\left(T^{*} M, P, \bar{g}\right)$ and $\left(T^{*} M, P_{1}, \bar{g}_{1}\right)$ are almost para-Kähler (resp. para-Kähler). Moreover, we establish that the defined almost para-complex structures $P$ and $P_{1}$ are harmonic with respect to $\bar{g}$ and $\bar{g}_{1}$, respectively. In Sect. 4 we study a family of non-degenerate hypersurfaces $\widetilde{H}_{t}$ of $\left(T^{*} M, P, \bar{g}\right)$. They are a generalization of the family $H_{t}$ of non-degenerate hypersurfaces of $\left(T^{*} M, \bar{g}\right)$, introduced in [3]. On a hypersurface $\widetilde{H}_{t}$ with a time-like unit normal vector field we define an almost paracontact metric structure $(\varphi, \bar{\xi}, \eta, g)$ induced from the almost para-Hermitian structure $(P, \bar{g})$. We determine the classes to which belong the obtained almost paracontact metric manifolds $\left(\widetilde{H}_{t}, \varphi, \bar{\xi}, \eta, g\right)$ and give a necessary and sufficient condition the considered manifolds to be paracontact metric. Also, we consider the almost paracontact metric manifolds $H_{t}$ and obtain a necessary and sufficient condition they to be para-Sasakian or K-paracontact metric.

\section{Preliminaries}

Let $M$ be a connected smooth $n$-dimensional manifold $(n \geq 2)$. The cotangent bundle $T^{*} M$ of $M$ consists of all pairs $(x, \omega)$, where $x \in M$ and $\omega \in T_{x}^{*} M$. Let $p: T^{*} M \longrightarrow M, \quad p(x, \omega)=x$, be the natural projection of $T^{*} M$ to $M$. Any local chart $\left(U ; x^{1}, \ldots, x^{n}\right)$ on $M$ induces a local chart $\left(p^{-1}(U) ; x^{1}, \ldots, x^{n}, x^{1 *}, \ldots, x^{n *}\right)$ on $T^{*} M$, where for any $i=1, \ldots, n$ the function $x^{i} \circ p$ on $p^{-1}(U)$ is identified with the function $x^{i}$ on $U$ and $x^{i *}=\omega_{i}=\omega\left(\left(\frac{\partial}{\partial x^{i}}\right)_{x}\right)$ at any point $(x, \omega) \in p^{-1}(U)$. The vectors $\left\{\left(\partial_{1}\right)_{(x, \omega)}, \ldots,\left(\partial_{n}\right)_{(x, \omega)},\left(\partial_{1 *}\right)_{(x, \omega)}, \ldots,\left(\partial_{n *}\right)_{(x, \omega)}\right\}$, where we put $\partial_{i}=\frac{\partial}{\partial x^{i}}$ and $\partial_{i *}=\frac{\partial}{\partial \omega^{i}}(i=1, \ldots, n)$ form a basis of the tangent space $\left(T^{*} M\right)_{(x, \omega)}$ at each point $(x, \omega) \in T^{*} M$. The Liouville type vector field $W$ is globally defined vector field on $T^{*} M$ which is expressed in local coordinates by

$$
W=\sum_{i=1}^{n} \omega_{i} \partial_{i *} .
$$

Everywhere here we will denote by $\mathcal{F}(M), \chi(M)$ and $\Omega^{1}(M)$ the set of all smooth real functions, vector fields and differential 1-forms on $M$, respectively.

Now, we recall the constructions of the vertical and complete lifts for which we refer to [10, 11]. 
The vertical lift $f^{V}$ on $T^{*} M$ of a function $f \in \mathcal{F}(M)$ is a function on $T^{*} M$ defined by $f^{V}=f \circ p$. The vertical lift $X^{V}$ on $T^{*} M$ of a vector field $X \in \chi(M)$ is a function on $T^{*} M$ (called evaluation function) defined by

$$
X^{V}(x, \omega)=\omega\left(X_{x}\right) \text { or equivalently } X^{V}(x, \omega)=\omega_{i} X^{i}(x) \text {, where } X=X^{i} \partial_{i} .
$$

In [11] it is shown that a vector field $U \in \chi\left(T^{*} M\right)$ is determined by its action on all evaluation functions. More precisely, the following proposition is valid:

Proposition 2.1. [11] Let $U_{1}$ and $U_{2}$ be vector fields on $T^{*} M$. If $U_{1}\left(Z^{V}\right)=U_{2}\left(Z^{V}\right)$ holds for all $Z \in \chi(M)$, then $U_{1}=U_{2}$.

The vertical lift $\alpha^{V}$ on $T^{*} M$ of a differential 1-form $\alpha \in \Omega^{1}(M)$ is a tangent vector field to $T^{*} M$ which is defined by

$$
\alpha^{V}\left(Z^{V}\right)=(\alpha(Z))^{V}, \quad Z \in \chi(M) .
$$

In local coordinates we have

$$
\alpha^{V}=\sum_{i=1}^{n} \alpha_{i} \partial_{i *}
$$

where $\alpha=\sum_{i=1}^{n} \alpha_{i} \mathrm{~d} x^{i}$. Hence, identifying $f^{V} \in \mathcal{F}\left(T^{*} M\right)$ with $f \in \mathcal{F}(M)$, we obtain $\alpha^{V}\left(f^{V}\right)=0$ for all $f \in \mathcal{F}(M)$.

The complete lift $X^{C}$ on $T^{*} M$ of a vector field $X \in \chi(M)$ is a tangent vector field to $T^{*} M$ which is defined by

$$
X^{C}\left(Z^{V}\right)=[X, Z]^{V}, \quad Z \in \chi(M) .
$$

In local coordinates $X^{C}$ is written as

$$
X_{(x, \omega)}^{C}=\sum_{i=1}^{n} X^{i}(x)\left(\partial_{i}\right)_{(x, \omega)}-\sum_{h, i=1}^{n} \omega_{h}\left(\partial_{i} X^{h}\right)(x)\left(\partial_{i *}\right)_{(x, \omega)},
$$

where $X=X^{i} \partial_{i}$. Therefore we have $X^{C}\left(f^{V}\right)=(X f)^{V}$ for all $f \in \mathcal{F}(M)$.

We note that the tangent space $T_{(x, \omega)} T^{*} M$ of $T^{*} M$ at any point $(x, \omega) \in T^{*} M$ is generated by the vector fields of the form $\alpha^{V}+X^{C}$.

\section{Almost para-Hermitian structures induced by natural Riemann EXTENSIONS}

This section deals with para-Hermitian geometry and first we will recall some basic notions.

An $(1,1)$ tensor field $P$ on a $2 n$-dimensional smooth manifold $N$ is said to be an almost product structure if $P \neq \pm \mathrm{Id}$ and $P^{2}=\mathrm{Id}$. In this case the pair $(N, P)$ is called an almost product manifold. An almost product structure $P$ on $N$ such that the eigendistributions of $P$ corresponding to the eigenvalues \pm 1 of $P$ have the same rank, is called a para-complex structure and $(N, P)$ - an almost para-complex manifold.

A $2 n$-dimensional smooth manifold $N$ has an almost para-Hermitian structure $(P, g)$ if it is endowed with an almost para-complex structure $P$ and a semi-Riemannian metric $g$ such that $P$ is an anti-isometry with respect to $g$, i.e. $g(P X, P Y)=$ 
$-g(X, Y), \quad X, Y \in \chi(N)$. The manifold $(N, P, g)$ is called an almost para-Hermitian manifold. The metric $g$ is necessarily indefinite of a neutral signature. The fundamental 2 -form $\Omega$ and the tensor field $F$ of type $(0,3)$ of an almost para-Hermitian manifold are defined by $\Omega(X, Y)=g(X, P Y)$ and $F(X, Y, Z)=g\left(\left(\nabla_{X} P\right) Y, Z\right)$, respectively, where $\nabla$ is the Levi-Civita connection of $g$. The tensor field $F$ has the following properties:

$$
F(X, Y, Z)=-F(X, Z, Y), \quad F(X, P Y, P Z)=F(X, Y, Z), X, Y, Z \in \chi(N) .
$$

A classification of the almost para-Hermitian manifolds is given in [1]. Here we recall the characteristic conditions of two basic classes of almost para-Hermitian manifolds:

- $(N, P, g)$ is para-Kähler if $\nabla P=0 \Longleftrightarrow F=0$;

- $(N, P, g)$ is almost para-Kähler if $\mathrm{d} \Omega(X, Y, Z)=0 \Longleftrightarrow$

$$
\underset{(X, Y, Z)}{\mathfrak{S}} F(X, Y, Z)=0 \text {, where } \underset{(X, Y, Z)}{\mathfrak{S}} \text { denotes the cyclic sum over } X, Y, Z .
$$

In this section we also need the following notion introduced in [7]:

Definition 3.1. Any $(1,1)$-tensor field $T$ on a (semi-) Riemannian manifold $(N, h)$ is called harmonic if $T$ viewed as an endomorphism field $T:\left(T N, h^{C}\right) \longrightarrow\left(T N, h^{C}\right)$ is a harmonic map, where $h^{C}$ denotes the complete lift (see [10]) of the (semi-) Riemannian metric $h$.

We recall

Proposition 3.2. 7] Let $(N, h)$ be a (semi-) Riemannian manifold and let $\nabla$ be the Levi-Civita connection of $h$. Then any $(1,1)$-tensor field $T$ on $(N, h)$ is harmonic if and only if $\delta T=0$, where

$$
\delta T=\operatorname{trace}_{h}(\nabla T)=\operatorname{trace}_{h}\left\{(X, Y) \longrightarrow\left(\nabla_{X} T\right) Y\right\} .
$$

Further, if it is not otherwise stated, we assume that $(M, \nabla)$ is an n-dimensional manifold endowed with a symmetric linear connection $\nabla$ (i. e. $\nabla$ is torsion-free). In [8] Sekizawa constructed a semi-Riemannian metric $\bar{g}$ at each point $(x, \omega)$ of the cotangent bundle $T^{*} M$ of $M$ by:

$$
\begin{aligned}
& \bar{g}_{(x, \omega)}\left(X^{C}, Y^{C}\right)=-a \omega\left(\nabla_{X_{x}} Y+\nabla_{Y_{x}} X\right)+b \omega\left(X_{x}\right) \omega\left(Y_{x}\right), \\
& \bar{g}_{(x, \omega)}\left(X^{C}, \alpha^{V}\right)=a \alpha_{x}\left(X_{x}\right), \\
& \bar{g}_{(x, \omega)}\left(\alpha^{V}, \beta^{V}\right)=0
\end{aligned}
$$

for all vector fields $X, Y$ and all differential 1-forms $\alpha, \beta$ on $M$, where $a, b$ are arbitrary constants. We may assume $a>0$ without loss of generality. The semiRiemannian metric $\bar{g}$ defined by (3.2) is called a natural Riemann extension [5, 8]. When $b \neq 0 \bar{g}$ is called a proper natural Riemann extension. In the case when $a=1$ and $b=0$ we obtain the notion of the classical Riemann extension defined by Patterson and Walker (see [6, 9]). In [3] it is shown that $\bar{g}$ is of neutral signature $(n, n)$.

In 22 authors have constructed a canonical almost para-complex structure $\mathcal{P}$ on $T^{*} M$ by $\mathcal{P} X^{C}=X^{C}$ and $\mathcal{P} \alpha^{V}=-\alpha^{V}$, where $X^{C}$ and $\alpha^{V}$ are the complete lift of a vector field $X$ and the vertical lift of a differential 1-form $\alpha$ on $M$, respectively. 
They proved that $\mathcal{P}$ is harmonic if and only if the natural Riemann extension $\bar{g}$ on $T^{*} M$ is non-proper.

In this section we shall construct almost para-complex structures $P$ and $P_{1}$ on $T^{*} M$ such that $(P, \bar{g})$ and $\left(P_{1}, \bar{g}_{1}\right)$ are almost para-Hermitian structures on $T^{*} M$, where $\bar{g}$ (resp. $\bar{g}_{1}$ ) is the proper (resp. non-proper) natural Riemann extension on $T^{*} M$. Moreover, we show that $P$ and $P_{1}$ are harmonic with respect to $\bar{g}$ and $\bar{g}_{1}$, respectively.

The following conventions and formulas will be used later on.

Let $T$ be an $(1,1)$ tensor field on a manifold $M$. Then the contracted vector field $C(T) \in \chi\left(T^{*} M\right)$ is defined at any point $(x, \omega) \in T^{*} M$ by its value on any evaluation function as follows:

$$
C(T)\left(Z^{V}\right)_{(x, \omega)}=(T Z)_{(x, \omega)}^{V}=\omega\left((T Z)_{x}\right), \quad Z \in \chi(M)
$$

For an 1 -form $\alpha$ on $M$ we denote by $i_{\alpha}(T)$ the 1 -form on $M$, defined by

$$
\left(i_{\alpha}(T)\right)(Z)=\alpha(T Z), \quad Z \in \chi(M) .
$$

By using (3.4) we obtain

$$
\left(i_{\alpha}(T)\right)^{V}(Z)_{(x, \omega)}^{V}=(\omega(T))^{V}(Z)_{(x, \omega)}^{V}=\omega\left((T Z)_{x}\right), \quad Z \in \chi(M)
$$

Now, the equalities (3.3), (3.5) and Proposition 2.1) imply that at each point $(x, \omega) \in$ $T^{*} M$ the following equality holds

$$
C(T)_{(x, \omega)}=\left(\omega_{x}(T)\right)^{V}
$$

Also, at each point $(x, \omega) \in T^{*} M$ we have

$$
W_{(x, \omega)}=\left(\omega_{x}\right)^{V}
$$

Taking into account (3.2), (3.6) and (3.7) we obtain

$$
\begin{aligned}
& \bar{g}_{(x, \omega)}\left(X^{C}, C(T)\right)=a \omega_{x}\left((T X)_{x}\right), \quad \bar{g}_{(x, \omega)}\left(W, \alpha^{V}\right)=0, \\
& \bar{g}_{(x, \omega)}(W, W)=\bar{g}_{(x, \omega)}(W, C(T))=\bar{g}_{(x, \omega)}\left(C\left(T_{1}\right), C\left(T_{2}\right)\right)=0,
\end{aligned}
$$

where $T_{1}$ and $T_{2}$ are arbitrary $(1,1)$ tensor fields on $M$. 
For the Levi-Civita connection $\bar{\nabla}$ of the proper natural Riemann extension $\bar{g}$ we get the formulas (see [5]):

$$
\begin{aligned}
&\left(\bar{\nabla}_{X^{C}} Y^{C}\right)_{(x, \omega)}=\left(\nabla_{X} Y\right)_{(x, \omega)}^{C}+C((\nabla X)(\nabla Y)+(\nabla Y)(\nabla X))_{(x, \omega)} \\
&+C(R(., X) Y+R(., Y) X)_{(x, \omega)} \\
&-\frac{b}{2 a}\left\{\omega(Y) X^{C}+\omega(X) Y^{C}+2 \omega(Y) C(\nabla X)+2 \omega(X) C(\nabla Y)\right. \\
&\left.+\omega\left(\nabla_{X} Y+\nabla_{Y} X\right) W\right\}_{(x, \omega)}+\frac{b^{2}}{a^{2}} \omega(X) \omega(Y) W_{(x, \omega)}, \\
&\left(\bar{\nabla}_{X^{C}} \beta^{V}\right)_{(x, \omega)}=\left(\nabla_{X} \beta\right)_{(x, \omega)}^{V}+\frac{b}{2 a}\left\{\omega(X) \beta^{V}+\beta(X) W\right\}_{(x, \omega)}, \\
&\left(\bar{\nabla}_{\alpha^{V}} Y^{C}\right)_{(x, \omega)}=-\left(i_{\alpha}(\nabla Y)\right)_{(x, \omega)}^{V}+\frac{b}{2 a}\left\{\omega(Y) \alpha^{V}+\alpha(Y) W\right\}_{(x, \omega)}, \\
&\left(\bar{\nabla}_{\alpha^{V}} \beta^{V}\right)_{(x, \omega)}=0, \quad\left(\bar{\nabla}_{X C} W\right)_{(x, \omega)}=-C(\nabla X)_{(x, \omega)}+\frac{b}{a} \omega(X) W_{(x, \omega)}, \\
&\left(\bar{\nabla}_{\alpha^{V}} W\right)_{(x, \omega)}=\alpha_{(x, \omega)}^{V}, \quad\left(\bar{\nabla}_{W} W\right)_{(x, \omega)}=W_{(x, \omega)},
\end{aligned}
$$

where: $X^{C}, Y^{C}$ and $\alpha^{V}, \beta^{V}$ are the complete lifts of the vector fields $X, Y \in \chi(M)$ and the vertical lifts of the differential 1-forms $\alpha, \beta$ on $M$, respectively; $C(\nabla X) \in$ $\chi\left(T^{*} M\right)$ is the contracted $(1,1)$ tensor field $\nabla X$ on $M$, defined by $(\nabla X)(Z)=\nabla_{Z} X$, $Z \in \chi(M) ; R$ is the curvature tensor of $\nabla$ and $C(R(., X) Y)$ is the contracted $(1,2)$ tensor field $R(., X) Y$ on $M$ given by $(R(., X) Y)(Z)=R(Z, X) Y), Z \in \chi(M)$.

On $T^{*} M$ endowed with a proper natural Riemann extension $\bar{g}$ we define the endomorphism $P$ by

$$
\begin{aligned}
& P X^{C}=X^{C}+2 C(\nabla X)-\frac{b}{a} X^{V} W, \\
& P \alpha^{V}=-\alpha^{V} .
\end{aligned}
$$

Theorem 3.3. Let the total space of the cotangent bundle $T^{*} M$ of an $n$-dimensional manifold $(M, \nabla)$ be endowed with the proper natural Riemann extension $\bar{g}$, defined by (3.2), and the endomorphism $P$, defined by (3.10). Then $\left(T^{*} M, P, \bar{g}\right)$ is an almost para-Hermitian manifold. Moreover

(i) if $M$ is not flat (resp. $M$ is flat), then $\left(T^{*} M, P, \bar{g}\right)$ is almost para-Kähler (resp. para-Kähler);

(ii) $P$ is harmonic on $\left(T^{*} M, \bar{g}\right)$.

Proof. From (3.6), (3.7) and (3.10) it follows that

$$
P(C(\nabla X))=-C(\nabla X), \quad P W=-W .
$$

By using (3.10) and (3.11) we see that $P \neq \mathrm{Id}$ and $P^{2}=\mathrm{Id}$. One can easily verify that the eigendistributions of $P$ corresponding to the eigenvalues \pm 1 of $P$ have the same rank. Hence, $P$ is an almost para-complex structure on $T^{*} M$. By direct 
calculations, using (3.2), (3.8) and (3.10) we obtain

$$
\begin{aligned}
& \bar{g}\left(P X^{C}, P Y^{C}\right)=-\bar{g}\left(X^{C}, Y^{C}\right), \quad \bar{g}\left(P X^{C}, P \alpha^{V}\right)=-\bar{g}\left(X^{C}, \alpha^{V}\right), \\
& \bar{g}\left(P \alpha^{V}, P \beta^{V}\right)=-\bar{g}\left(\alpha^{V}, \beta^{V}\right),
\end{aligned}
$$

which means that $\left(T^{*} M, P, \bar{g}\right)$ is an almost para-Hermitian manifold.

(i) Further, we find the tensor field $\bar{F}(\bar{X}, \bar{Y}, \bar{Z})=\bar{g}\left(\left(\bar{\nabla}_{\bar{X}} P\right) \bar{Y}, \bar{Z}\right)$ on $\left(T^{*} M, P, \bar{g}\right)$, where $\bar{X}, \bar{Y}, \bar{Z} \in \chi\left(T^{*} M\right)$. By using (3.2), (3.8), (3.9), (3.10) and (3.11) we obtain

$$
\begin{aligned}
& \bar{F}_{(x, \omega)}\left(X^{C}, Y^{C}, Z^{C}\right)=2 a \omega\left(R_{x}(Z, Y) X\right), \\
& \bar{F}_{(x, \omega)}\left(X^{C}, \alpha^{V}, Z^{C}\right)=-\bar{F}_{(x, \omega)}\left(X^{C}, Z^{C}, \alpha^{V}\right)=0, \\
& \bar{F}_{(x, \omega)}\left(\alpha^{V}, \beta^{V}, Z^{C}\right)=-\bar{F}_{(x, \omega)}\left(\alpha^{V}, Z^{C}, \beta^{V}\right)=0, \\
& \bar{F}_{(x, \omega)}\left(\alpha^{V}, Y^{C}, Z^{C}\right)=\bar{F}_{(x, \omega)}\left(X^{C}, \beta^{V}, \gamma^{V}\right)=\bar{F}_{(x, \omega)}\left(\alpha^{V}, \beta^{V}, \gamma^{V}\right)=0 .
\end{aligned}
$$

If $M$ is flat, then from (3.12) it follows that $\bar{F}\left(X^{C}+\alpha^{V}, Y^{C}+\beta^{V}, Z^{C}+\gamma^{V}\right)=0$ for arbitrary $X^{C}+\alpha^{V}, Y^{C}+\beta^{V}, Z^{C}+\gamma^{V} \in \chi\left(T^{*} M\right)$ which means that $\left(T^{*} M, P, \bar{g}\right)$ is para-Kähler. In the case when $M$ is not flat, then the equalities (3.12) and the first identity of Bianchi for $R$ imply

$$
\begin{aligned}
& \underset{\left(X^{C}+\alpha^{V}, Y^{C}+\beta^{V}, Z^{C}+\gamma^{V}\right)}{\mathfrak{F}} \bar{F}\left(X^{C}+\alpha^{V}, Y^{C}+\beta^{V}, Z^{C}+\gamma^{V}\right)= \\
& \underset{\left(X^{C}, Y^{C}, Z^{C}\right)}{F}\left(X^{C}, Y^{C}, Z^{C}\right)=2 a \underset{(X, Y, Z)}{\mathfrak{S}} \omega(R(Z, Y) X)=0,
\end{aligned}
$$

i.e. $\left(T^{*} M, P, \bar{g}\right)$ is an almost para-Kähler manifold.

(ii) As an consequence from the characteristic condition $\underset{(X, Y, Z)}{\mathfrak{S}} F(X, Y, Z)=0$ of an almost para-Kähler manifold $(N, P, g)$ and the properties (3.1) of $F$ we obtain

$$
F(P X, P Y, Z)=F(X, Y, Z), \quad X, Y, Z \in \chi(N) .
$$

The last equality implies $\left(\nabla_{X} P\right) Y=\left(\nabla_{P X} P\right) P Y$. Then if $\left\{e_{1}, \ldots, e_{n}, P e_{1}, \ldots, P e_{n}\right\}$ is an orthonormal basis on $N$, such that $g\left(e_{i}, e_{i}\right)=-g\left(P e_{i}, P e_{i}\right)=1(i=1, \ldots, n)$, for $\delta P$ we have

$$
\delta P=\operatorname{trace}_{g} \nabla P=\sum_{i=1}^{n}\left\{\left(\nabla_{e_{i}} P\right) e_{i}-\left(\nabla_{P e_{i}} P\right) P e_{i}\right\}=0 .
$$

Hence, the almost para-complex structure $P$ is harmonic on every almost paraKähler manifold $(N, P, g)$. In the case when $(N, P, g)$ is para-Kähler, then $\nabla P=0$ and $\delta P=0$ holds too.

Now, let us assume that $T^{*} M$ is endowed with a non-proper natural Riemann extension $\bar{g}_{1}$, i.e. $\bar{g}_{1}$ is given by (3.2) and $b=0$. We define the endomorphism $P_{1}$ by

$$
\begin{aligned}
& P_{1} X^{C}=X^{C}+2 C(\nabla X), \\
& P_{1} \alpha^{V}=-\alpha^{V} .
\end{aligned}
$$

By direct verification we establish that $\left(P_{1}, \bar{g}_{1}\right)$ is an almost para-Hermitian structure on $T^{*} M$ which is obtained from the almost para-Hermitian structure $(P, \bar{g})$ on $T^{*} M$ by $b=0$. Moreover, from (3.12) we see that the tensor $\bar{F}$ on $\left(T^{*} M, P, \bar{g}\right)$ does not depend on $b$. Therefore we obtain 
Theorem 3.4. Let the total space of the cotangent bundle $T^{*} M$ of an $n$-dimensional manifold $(M, \nabla)$ be endowed with the non-proper natural Riemann extension $\bar{g}_{1}$ and the endomorphism $P_{1}$, defined by (3.14). Then $\left(T^{*} M, P_{1}, \bar{g}_{1}\right)$ is an almost paraHermitian manifold. Moreover

(i) if $M$ is not flat (resp. $M$ is flat), then $\left(T^{*} M, P_{1}, \bar{g}_{1}\right)$ is almost para-Kähler (resp. para-Kähler);

(ii) $P_{1}$ is harmonic on $\left(T^{*} M, \bar{g}_{1}\right)$.

\section{Almost paracontact metric structures induCed by Proper natural RIEMANN EXTENSIONS}

In this section we will construct almost paracontact metric structures on hypersurfaces of almost para-Kähler and para-Kähler manifolds $\left(T^{*} M, P, \bar{g}\right)$ considered in \$.

A $(2 \mathrm{n}+1)$-dimensional smooth manifold $\widetilde{M}$ has an almost paracontact structure $(\varphi, \bar{\xi}, \eta)$ if it admits a tensor field $\varphi$ of type $(1,1)$, a vector field $\bar{\xi}$ and a 1 -form $\eta$ satisfying the following conditions:

$$
\varphi^{2}=\mathrm{Id}-\eta \otimes \bar{\xi}, \quad \eta(\bar{\xi})=1, \quad \varphi(\bar{\xi})=0 .
$$

As immediate consequences of the definition of the almost paracontact structure we have that the endomorphism $\varphi$ has $\operatorname{rank} 2 n$ and $\eta \circ \varphi=0$. If a manifold $\widetilde{M}$ with $(\varphi, \bar{\xi}, \eta)$-structure admits a pseudo-Riemannian metric $g$ such that

$$
g(\varphi X, \varphi Y)=-g(X, Y)+\eta(X) \eta(Y), \quad X, Y \in \chi(\widetilde{M})
$$

then we say that $\widetilde{M}$ has an almost paracontact metric structure and $(\widetilde{M}, \varphi, \bar{\xi}, \eta, g)$ is called an almost paracontact metric manifold. The metric $g$ is called compatible metric and it is necessarily of signature $(n+1, n)$. Setting $Y=\bar{\xi}$, we have $\eta(X)=$ $g(X, \bar{\xi})$.

The fundamental 2-form $\phi$ on $(\widetilde{M}, \varphi, \bar{\xi}, \eta, g)$ is given by $\phi(X, Y)=g(X, \varphi Y)$ and the tensor field $\widetilde{F}$ of type $(0,3)$ is defined by

$$
\widetilde{F}(X, Y, Z)=(\widetilde{\nabla} \phi)(X, Y, Z)=\left(\widetilde{\nabla}_{X} \phi\right)(Y, Z)=g\left(\left(\widetilde{\nabla}_{X} \varphi\right) Y, Z\right),
$$

where $X, Y, Z \in \chi(\widetilde{M})$ and $\widetilde{\nabla}$ is the Levi-Civita connection on $\widetilde{M}$. The tensor field $\widetilde{F}$ has the following properties:

$$
\begin{aligned}
& \widetilde{F}(X, Y, Z)=-\widetilde{F}(X, Z, Y), \\
& \widetilde{F}(X, \varphi Y, \varphi Z)=\widetilde{F}(X, Y, Z)+\eta(Y) \widetilde{F}(X, Z, \bar{\xi})-\eta(Z) \widetilde{F}(X, Y, \bar{\xi}) .
\end{aligned}
$$

The following 1-forms are associated with $\widetilde{F}$ :

$$
\theta(X)=g^{i j} \widetilde{F}\left(e_{i}, e_{j}, X\right) ; \theta^{*}(X)=g^{i j} \widetilde{F}\left(e_{i}, \varphi e_{j}, X\right) ; \omega(X)=\widetilde{F}(\bar{\xi}, \bar{\xi}, X),
$$

where $\left\{e_{i}, \bar{\xi}\right\}(i=1, \ldots, 2 n)$ is a basis of $T \widetilde{M}$, and $\left(g^{i j}\right)$ is the inverse matrix of $\left(g_{i j}\right)$. An almost paracontact metric manifold is called

- normal if $N(X, Y)-2 d \eta(X, Y) \bar{\xi}=0$, where

$$
N(X, Y)=\varphi^{2}[X, Y]+[\varphi X, \varphi Y]-\varphi[\varphi X, Y]-\varphi[X, \varphi Y]
$$


is the Nijenhuis torsion tensor of $\varphi$ (see []);

- paracontact metric if $\phi=d \eta$;

- $\alpha$-para-Sasakian if $\left(\widetilde{\nabla}_{X} \varphi\right) Y=\alpha(g(X, Y) \bar{\xi}-\eta(Y) X)$, where $\alpha \neq 0$ is constant;

- para-Sasakian if it is normal and paracontact metric;

- $\alpha$-para-Kenmotsu if $\left(\widetilde{\nabla}_{X} \varphi\right) Y=-\alpha(g(X, \varphi Y) \bar{\xi}+\eta(Y) \varphi X)$, where $\alpha \neq 0$ is constant, in particular, para-Kenmotsu if $\alpha=-1$;

- K-paracontact if it is paracontact and $\bar{\xi}$ is Killing vector field;

- quasi-para-Sasakian if it is normal and $d \phi=0$.

Twelve basic classes of almost paracontact metric manifolds with respect to the tensor field $\widetilde{F}$ were obtained in [12]. Further we give the characteristic conditions of these classes:

$$
\begin{aligned}
& \mathbb{G}_{1}: \widetilde{F}(X, Y, Z)=\frac{1}{2(n-1)}\{g(X, \varphi Y) \theta(\varphi Z)-g(X, \varphi Z) \theta(\varphi Y) \\
& \left.-g(\varphi X, \varphi Y) \theta\left(\varphi^{2} Z\right)+g(\varphi X, \varphi Z) \theta\left(\varphi^{2} Y\right)\right\}, \\
& \mathbb{G}_{2}: \widetilde{F}(\varphi X, \varphi Y, Z)=-\widetilde{F}(X, Y, Z), \quad \theta=0, \\
& \mathbb{G}_{3}: \widetilde{F}(\bar{\xi}, Y, Z)=\widetilde{F}(X, \bar{\xi}, Z)=0, \quad \widetilde{F}(X, Y, Z)=-\widetilde{F}(Y, X, Z), \\
& \mathbb{G}_{4}: \widetilde{F}(\bar{\xi}, Y, Z)=\widetilde{F}(X, \bar{\xi}, Z)=0, \quad \underset{(X, Y, Z)}{\mathfrak{S}} \widetilde{F}(X, Y, Z)=0, \\
& \mathbb{G}_{5}: \tilde{F}(X, Y, Z)=\frac{\theta(\bar{\xi})}{2 n}\{\eta(Y) g(\varphi X, \varphi Z)-\eta(Z) g(\varphi X, \varphi Y)\}, \\
& \mathbb{G}_{6}: \widetilde{F}(X, Y, Z)=-\frac{\theta^{*}(\bar{\xi})}{2 n}\{\eta(Y) g(X, \varphi Z)-\eta(Z) g(X, \varphi Y)\}, \\
& \mathbb{G}_{7}: \widetilde{F}(X, Y, Z)=-\eta(Y) \widetilde{F}(X, Z, \bar{\xi})+\eta(Z) \widetilde{F}(X, Y, \bar{\xi}), \\
& \widetilde{F}(X, Y, \bar{\xi})=-\widetilde{F}(Y, X, \bar{\xi})=-\widetilde{F}(\varphi X, \varphi Y, \bar{\xi}), \quad \theta^{*}(\bar{\xi})=0, \\
& \mathbb{G}_{8}: \widetilde{F}(X, Y, Z)=-\eta(Y) \widetilde{F}(X, Z, \bar{\xi})+\eta(Z) \widetilde{F}(X, Y, \bar{\xi}), \\
& \widetilde{F}(X, Y, \bar{\xi})=\widetilde{F}(Y, X, \bar{\xi})=-\widetilde{F}(\varphi X, \varphi Y, \bar{\xi}), \quad \theta(\bar{\xi})=0, \\
& \mathbb{G}_{9}: \widetilde{F}(X, Y, Z)=-\eta(Y) \widetilde{F}(X, Z, \bar{\xi})+\eta(Z) \widetilde{F}(X, Y, \bar{\xi}), \\
& \widetilde{F}(X, Y, \bar{\xi})=-\widetilde{F}(Y, X, \bar{\xi})=\widetilde{F}(\varphi X, \varphi Y, \bar{\xi}), \\
& \mathbb{G}_{10}: \widetilde{F}(X, Y, Z)=-\eta(Y) \widetilde{F}(X, Z, \bar{\xi})+\eta(Z) \widetilde{F}(X, Y, \bar{\xi}), \\
& \widetilde{F}(X, Y, \bar{\xi})=\widetilde{F}(Y, X, \bar{\xi})=\widetilde{F}(\varphi X, \varphi Y, \bar{\xi}),
\end{aligned}
$$




$$
\begin{gathered}
\mathbb{G}_{11}: \widetilde{F}(X, Y, Z)=\eta(X) \widetilde{F}(\bar{\xi}, \varphi Y, \varphi Z), \\
\mathbb{G}_{12}: \widetilde{F}(X, Y, Z)=\eta(X)\{\eta(Y) \widetilde{F}(\bar{\xi}, \bar{\xi}, Z)-\eta(Z) \widetilde{F}(\bar{\xi}, \bar{\xi}, Y)\} .
\end{gathered}
$$

In 12 the classes of $\alpha$-para-Sasakian, $\alpha$-para-Kenmotsu, normal, paracontact metric, para-Sasakian, K-paracontact and quasi-para-Sasakian manifolds are determined. Also, the classes of the 3-dimensional almost paracontact metric manifolds are obtained. Here, we recall some of the theorems in [12] which we need.

Let $\overline{\mathbb{G}}_{5}$ be the subclass of $\mathbb{G}_{5}$ which consists of all $(2 n+1)$-dimensional $\mathbb{G}_{5}$ manifolds such that $\theta(\xi)=2 n$ (resp. $\theta(\xi)=-2 n)$ by $\phi(X, Y)=g(\varphi X, Y)$ (resp. $\phi(X, Y)=g(X, \varphi Y))$.

Theorem 4.1. 12] A $(2 n+1)$-dimensional almost paracontact metric manifold $(\widetilde{M}, \varphi, \bar{\xi}$, $\eta, g)$ is:

(i) paracontact metric if and only if $\widetilde{M}$ belongs to the class $\overline{\mathbb{G}}_{5}$ or to the classes which are direct sums of $\overline{\mathbb{G}}_{5}$ with $\mathbb{G}_{4}$ and $\mathbb{G}_{10}$;

(ii) para-Sasakian if and only if $\widetilde{M}$ belongs to the class $\overline{\mathbb{G}}_{5}$;

(iii) K-paracontact metric if and only if $\widetilde{M}$ belongs to the classes $\overline{\mathbb{G}}_{5}$ and $\overline{\mathbb{G}}_{5} \oplus \mathbb{G}_{4}$;

(iv) quasi-para-Sasakian if and only if $\widetilde{M}$ belongs to the classes $\mathbb{G}_{5}, \mathbb{G}_{8}$ and $\mathbb{G}_{5} \oplus \mathbb{G}_{8}$.

Proposition 4.2. 12 The 3-dimensional almost paracontact metric manifolds belong to the classes $\mathbb{G}_{5}, \mathbb{G}_{6}, \mathbb{G}_{10}, \mathbb{G}_{12}$ and to the classes which are their direct sums.

Let $(\bar{M}, P, \bar{g})$ be a $2 n$-dimensional almost para-Hermitian manifold and $\widetilde{M}$ be a $(2 n-1)$-dimensional differentiable hypersurface embeding in $\bar{M}$ such that the normal vector field $N$ to $\widetilde{M}$ is a time-like unit, i.e. $\bar{g}(N, N)=-1$. Hence, $P N$ is a space-like unit tangent vector field on $\widetilde{M}$. We denote the tangent and the normal component of the transform vector field $P X$ of an arbitrary tangent vector field $X \in \chi(\widetilde{M})$ by $\varphi X$ and $\eta(X) N$, respectively. Then $P X \in \chi(\widetilde{M})$ has the unique decomposition $P X=\varphi X+\eta(X) N$, where $\varphi$ is an $(1,1)$ tensor field on $\widetilde{M}$. The 1 -form $\eta$ on $\widetilde{M}$ is defined by $\eta(X)=\bar{g}(X, P N)$. So, at every point $p \in \widetilde{M}$ is determined the structure $(\varphi, \bar{\xi}, \eta, g)$, where

$$
\varphi X=P X-\eta(X) N, \quad \bar{\xi}=P N, \quad \eta(X)=\bar{g}(X, P N), \quad X \in \chi(\widetilde{M})
$$

and by $g$ is denoted the restriction of $\bar{g}$ on $\widetilde{M}$. It is easy to check that $(\varphi, \bar{\xi}, \eta, g)$ is an almost paracontact metric structure on $\widetilde{M}$, i.e. $(\widetilde{M}, \varphi, \bar{\xi}, \eta, g)$ is a $(2 n-1)$ dimensional almost paracontact metric manifold.

Let $\bar{\nabla}$ and $\widetilde{\nabla}$ be the Levi-Civita connections of the metrics $\bar{g}$ and $g$ on $\bar{M}$ and $\widetilde{M}$, respectively. Then the formulas of Gauss and Weingarten are:

$$
\bar{\nabla}_{X} Y=\widetilde{\nabla}_{X} Y-g\left(A_{N} X, Y\right) N, \quad \bar{\nabla}_{X} N=-A_{N} X, \quad X, Y \in \chi(\widetilde{M}),
$$

where $A_{N}$ is the second fundamental tensor of $\widetilde{M}$ corresponding to $N$. 
Using (4.27) and (4.28) we obtain

$$
\begin{gathered}
\bar{F}(X, Y, Z)=\widetilde{F}(X, Y, Z)-\eta(Y) g\left(A_{N} X, Z\right)+\eta(Z) g\left(A_{N} X, Y\right), \\
\bar{F}(X, Y, N)=\widetilde{F}(X, \varphi Y, \bar{\xi})+g\left(A_{N} X, \varphi Y\right),
\end{gathered}
$$

where $X, Y, Z \in \chi(\widetilde{M})$ and $\bar{F}, \widetilde{F}$ are the tensor fields on $\bar{M}$ and $\widetilde{M}$, defined by $\bar{F}(X, Y, Z)=\bar{g}\left(\left(\bar{\nabla}_{X} P\right) Y, Z\right), \quad \widetilde{F}(X, Y, Z)=g\left(\left(\widetilde{\nabla}_{X} \varphi\right) Y, Z\right)$, respectively. Let us assume that the $n$-dimensional manifold $M$ is endowed with both a symmetric linear connection $\nabla$ and with a globally defined nowhere zero vector field $\xi$ which is parallel with respect to $\nabla$, i.e. $\nabla \xi=0$ and $f$ is a function on $M$.

We consider the function $\tilde{f}: T^{*} M \longrightarrow \mathbb{R}$ defined by

$$
\tilde{f}=\xi^{V}+f^{V},
$$

or equivalently by $\widetilde{f}(x, \omega)=\omega_{x}\left(\xi_{x}\right)+f(x)$ for any $(x, \omega) \in T^{*} M$.

Let

$$
\widetilde{H}_{t}=\widetilde{f}^{-1}(t)=\left\{(x, \omega) \in T^{*} M: \widetilde{f}(x, \omega)=t, t \in \mathbb{R}\right\}
$$

be the hypersurfaces level set in $T^{*} M$, endowed with the restriction $g$ of the proper natural Riemann extension $\bar{g}$ on $T^{*} M$, where $f(x) \neq t$ at any point $x$ in $M$.

For later use, we recall that the gradient of a real function $F: N \longrightarrow \mathbb{R}$ on a (semi-) Riemannian manifold $(N, h)$ is given by $h(\operatorname{grad} F, X)=\mathrm{d} F(X), X \in \chi(N)$ and $h$ is a (semi-) Riemannian metric on $N$. In [4 the following formula for the gradient of the vertical lift $Z^{V}$ on $T^{*} M$ of $Z \in \chi(M)$ with respect to the proper natural Riemann extension $\bar{g}$ on $T^{*} M$ is obtained:

$$
\operatorname{grad} Z^{V}=\frac{1}{a}\left\{Z^{C}+2 C(\nabla Z)-\frac{b}{a} Z^{V} W\right\} .
$$

Theorem 4.3. Let $(M, \nabla)$ be a manifold endowed with a symmetric linear connection $\nabla$ inducing the proper natural Riemann extension $\bar{g}$ on $T^{*} M$ and $f$ be a function on $M$. If $t \in \mathbb{R}$ and $f(x) \neq t$ at any point $x$ in $M$, then:

(i) At any point $(x, \omega)$ of $\widetilde{H}_{t}$ the gradient of the function $\widetilde{f}$ is a normal vector field to $\widetilde{H}_{t}$ and it is given by

$$
\operatorname{grad} \tilde{f}=\frac{1}{a}\left\{\xi^{C}-\frac{b}{a} \xi^{V} W+(\mathrm{d} f)^{V}\right\} .
$$

(ii) The restriction $g$ of $\bar{g}$ on $\widetilde{H}_{t}$ is non-degenerate on $\widetilde{H}_{t}$, i.e. $\left(\widetilde{H}_{t}, g\right)$ is a semiRiemannian hypersurface of $T^{*} M$.

(iii) The vertical lift $\alpha^{V}$ of an 1-form $\alpha$ on $M$ and the complete lift $X^{C}$ of $X \in$ $\chi(M)$ are tangent to $\widetilde{H}_{t}$ if at any point $(x, \omega) \in \widetilde{H}_{t}$ they satisfy the conditions:

$$
\alpha_{x}\left(\xi_{x}\right)=0, \quad(X f)(x)=\omega_{x}\left(\left(\nabla_{\xi} X\right)_{x}\right) .
$$

Proof. (i) By using $\bar{g}(\operatorname{grad} \widetilde{f}, \bar{U})=(\mathrm{d} \widetilde{f})(\bar{U})$ for any tangent vector field $\bar{U}$ on $T^{*} M$ and $\widetilde{f}(x, \omega)=t \in \mathbb{R}$ at any point $(x, \omega) \in \widetilde{H}_{t}$, we obtain that $\bar{g}(\operatorname{grad} \widetilde{f}, U)=0$ for any vector field $U$ on $\widetilde{H}_{t}$. Therefore, $\operatorname{grad} \widetilde{f}$ is a normal vector field to $\widetilde{H}_{t}$. 
From the definition of the function $\tilde{f}$ it follows that $\operatorname{grad} \tilde{f}=\operatorname{grad} \xi^{V}+\operatorname{grad} f^{V}$. For $\operatorname{grad} \xi^{V}$, using (4.31) and taking into account that $\nabla \xi=0$, we have

$$
\operatorname{grad} \xi^{V}=\frac{1}{a}\left\{\xi^{C}-\frac{b}{a} \xi^{V} W\right\}
$$

Now, let us assume that $\operatorname{grad} f^{V}=Y^{C}+\theta^{V}$, where $Y \in \chi(M)$ and $\theta$ is an 1-form on $M$. Substituting $\operatorname{grad} f^{V}=Y^{C}+\theta^{V}$ in the equality $\bar{g}\left(\operatorname{grad} f^{V}, \alpha^{V}\right)=\alpha^{V}\left(f^{V}\right)=0$ we obtain $a \alpha(Y)=0$ for any 1-form $\alpha$ on $M$, which implies $Y=0$. Then from $\bar{g}\left(\operatorname{grad} f^{V}, X^{C}\right)=\bar{g}\left(\theta^{V}, X^{C}\right)=a \theta(X)$ and $\bar{g}\left(\operatorname{grad} f^{V}, X^{C}\right)=X^{C}\left(f^{V}\right)=(X f)^{V}=$ $((\mathrm{d} f) X)^{V}$ it follows that $\theta=\frac{1}{a} \mathrm{~d} f$. Hence, $\operatorname{grad} f^{V}=\frac{1}{a}(\mathrm{~d} f)^{V}$ and (4.32) holds.

(ii) For the normal vector field $\operatorname{grad} \widetilde{f}$ to $\widetilde{H}_{t}$ we compute $\bar{g}(\operatorname{grad} \tilde{f}, \operatorname{grad} \widetilde{f})=$ $-\frac{b(\omega(\xi))^{2}}{a^{2}}$, which shows that $\operatorname{grad} \tilde{f}$ is time-like or space-like when $b>0$ or $b<0$, respectively. Consequently, (ii) is proved.

(iii) $\alpha^{V}$ and $X^{C}$ are tangent to $\widetilde{H}_{t}$ if at any point $(x, \omega) \in \widetilde{H}_{t} \bar{g}\left(\operatorname{grad} \widetilde{f}, X^{C}\right)=$ $\bar{g}\left(\operatorname{grad} \tilde{f}, \alpha^{V}\right)=0$. By using (4.32) we obtain the equalities in (4.33).

Further, we consider a hypersurface $\widetilde{H}_{t}$ of $\left(T^{*} M, P, \bar{g}\right)$ with a time-like unit normal vector field $N$. According to Theorem 4.3, grad $\widetilde{f}$ is a normal vector field to $\widetilde{H}_{t}$ and it is time-like if $b>0$. Hence,

$$
N=\frac{1}{\sqrt{b} \xi^{V}}\left\{\xi^{C}-\frac{b}{a} \xi^{V} W+(\mathrm{d} f)^{V}\right\}
$$

Supplying $\widetilde{H}_{t}$ with the almost paracontact metric structure defined by (4.27), we have:

$$
\begin{aligned}
& \bar{\xi}=\frac{1}{\sqrt{b} \xi^{V}}\left\{\xi^{C}-(\mathrm{d} f)^{V}\right\}, \quad \eta\left(X^{C}\right)=-\frac{2 a}{\sqrt{b} \xi^{V}}(X f)^{V}+\sqrt{b} X^{V} \\
& \varphi X^{C}=X^{C}+2 C(\nabla X)-\frac{2 a}{\sqrt{b} \xi^{V}}(X f)^{V} W-\eta\left(X^{C}\right) \frac{1}{\sqrt{b} \xi^{V}}\left\{\xi^{C}+(\mathrm{d} f)^{V}\right\} \\
& \eta\left(\alpha^{V}\right)=0, \quad \varphi \alpha^{V}=-\alpha^{V} .
\end{aligned}
$$

Theorem 4.4. For the $(2 n-1)$-dimensional almost paracontact metric manifold $\left(\widetilde{H}_{t}, \varphi, \bar{\xi}, \eta, g\right)$ of $\left(T^{*} M, P, \bar{g}\right)$ with a time-like unit normal vector field $N$ and an almost paracontact metric structure given by (4.34) and (4.35), respectively, we have:

(i) If $M$ is flat or $\operatorname{dim} M=2$, then $\widetilde{H}_{t} \in \mathbb{G}_{5} \oplus \mathbb{G}_{10}$.

(ii) If $M$ is not flat and $\operatorname{dim} M>2$, then $\widetilde{H}_{t} \in \mathbb{G}_{4} \oplus \mathbb{G}_{5} \oplus \mathbb{G}_{10}$.

In both cases (i) and (ii) $\widetilde{H}_{t}$ is paracontact metric if and only if $b=4 a^{2}$.

Proof. From (4.29) for the tensor $\widetilde{F}$ on $\widetilde{H}_{t}$ we have

$$
\widetilde{F}(\widetilde{X}, \widetilde{Y}, \widetilde{Z})=\bar{F}(\widetilde{X}, \widetilde{Y}, \widetilde{Z})+\eta(\widetilde{Y}) g\left(A_{N} \widetilde{X}, \widetilde{Z}\right)-\eta(\widetilde{Z}) g\left(A_{N} \widetilde{X}, \widetilde{Y}\right)
$$


where $\widetilde{X}, \widetilde{Y}, \widetilde{Z} \in \chi\left(\widetilde{H}_{t}\right)$.

For arbitrary $X^{C} \in \chi\left(\widetilde{H}_{t}\right)$ and $\alpha^{V} \in \chi\left(\widetilde{H}_{t}\right)$, using (3.9), we find

$$
\begin{aligned}
A_{N} X^{C}=-\bar{\nabla}_{X^{C}} N & =-\frac{1}{\sqrt{b} \xi^{V}}\left\{C(R(., \xi) X)+\left(\nabla_{X} \mathrm{~d} f\right)^{V}\right\} \\
& +\frac{\sqrt{b}}{2 a}\left\{X^{C}+\eta\left(X^{C}\right) \bar{\xi}\right\}-\frac{2\left(\nabla_{\xi} X\right)^{V}}{\sqrt{b}\left(\xi^{V}\right)^{2}}(\mathrm{~d} f)^{V} \\
A_{N} \alpha^{V} & =-\bar{\nabla}_{\alpha^{V}} N=\frac{\sqrt{b}}{2 a} \alpha^{V} .
\end{aligned}
$$

Next, we calculate

$$
\begin{aligned}
& \bar{g}_{(x, \omega)}\left(A_{N} X^{C}, Z^{C}\right)=-\frac{a}{\sqrt{b} \omega(\xi)}\{\omega(R(Z, \xi) X)+X(Z f) \\
& \left.-\left(\nabla_{X} Z\right)(f)\right\}_{(x, \omega)}+\frac{\sqrt{b}}{2 a}\left\{\bar{g}\left(X^{C}, Z^{C}\right)+\eta\left(X^{C}\right) \eta\left(Z^{C}\right)\right\}_{(x, \omega)} \\
& -\left\{\frac{2 a \omega\left(\nabla_{\xi} X\right)}{\sqrt{b}(\omega(\xi))^{2}}(Z f)\right\}_{(x, \omega)} .
\end{aligned}
$$

From the first identity of Bianchi and $\nabla \xi=0$ we get

$$
R(Z, \xi) X=R(X, \xi) Z, \quad X, \xi, Z \in \chi(M) .
$$

Since $C(\nabla Z)$ is a vertical vector field on $T^{*} M$ and for $X^{C} \in \chi\left(\widetilde{H}_{t}\right)$ the following equality

$$
\left(\nabla_{\xi} X\right)^{V}=(X f)^{V}
$$

holds, we obtain

$$
\omega_{x}\left(\left(\nabla_{\nabla_{\xi} X} Z\right)_{x}\right)=C(\nabla Z)\left(\left(\nabla_{\xi} X\right)^{V}\right)_{(x, \omega)}=C(\nabla Z)\left((X f)^{V}\right)_{(x, \omega)}=0 .
$$

The equalities (4.40) and (4.42) imply

$$
\omega_{x}\left(R_{x}(Z, \xi) X\right)=\omega_{x}\left(\left(\nabla_{X} \nabla_{\xi} Z\right)_{x}\right)-\omega_{x}\left(\left(\nabla_{\xi} \nabla_{X} Z\right)_{x}\right) .
$$

By using (4.41) and (4.42) we get

$$
\begin{aligned}
(X(Z f))_{x} & =X^{C}\left((Z f)^{V}\right)_{(x, \omega)}=X^{C}\left(\left(\nabla_{\xi} Z\right)^{V}\right)_{(x, \omega)}=\left[X, \nabla_{\xi} Z\right]_{(x, \omega)}^{V} \\
& =\omega_{x}\left(\left(\nabla_{X} \nabla_{\xi} Z\right)_{x}\right) .
\end{aligned}
$$

Now, we substitute (4.44) and $\left(\left(\nabla_{X} Z\right)(f)\right)_{x}=\omega_{x}\left(\left(\nabla_{\xi} \nabla_{X} Z\right)_{x}\right)$ in (4.39). Then, taking into account (4.33) and (4.43), the equality (4.39) becomes

$$
\begin{aligned}
\bar{g}_{(x, \omega)}\left(A_{N} X^{C}, Z^{C}\right) & =-\frac{2 a}{\sqrt{b} \omega(\xi)}\{\omega(R(Z, \xi) X)\}_{(x, \omega)} \\
& +\frac{\sqrt{b}}{2 a}\left\{\bar{g}\left(X^{C}, Z^{C}\right)+\eta\left(X^{C}\right) \eta\left(Z^{C}\right)\right\}_{(x, \omega)} \\
& -\left\{\frac{2 a}{\sqrt{b}(\omega(\xi))^{2}}(X f)(Z f)\right\}_{(x, \omega)} .
\end{aligned}
$$


By using (3.12), (4.36) and the above equality we obtain

$$
\begin{aligned}
& \widetilde{F}_{(x, \omega)}\left(X^{C}, Y^{C}, Z^{C}\right)=\frac{2 a}{\sqrt{b} \omega(\xi)}\{\sqrt{b} \omega(\xi) \omega(R(Z, Y) X) \\
& \left.-\omega(R(Z, \xi) X) \eta\left(Y^{C}\right)+\omega(R(Y, \xi) X) \eta\left(Z^{C}\right)\right\}_{(x, \omega)} \\
& +\frac{\sqrt{b}}{2 a}\left\{\bar{g}\left(X^{C}, Z^{C}\right) \eta\left(Y^{C}\right)-\bar{g}\left(X^{C}, Y^{C}\right) \eta\left(Z^{C}\right)\right\}_{(x, \omega)} \\
& +\frac{2 a}{\sqrt{b}(\omega(\xi))^{2}}\left\{-(X f)(Z f) \eta\left(Y^{C}\right)+(X f)(Y f) \eta\left(Z^{C}\right)\right\}_{(x, \omega)} .
\end{aligned}
$$

After standard calculations, using (4.37) and (4.38), we find

$$
\begin{aligned}
& \widetilde{F}_{(x, \omega)}\left(X^{C}, Y^{C}, \gamma^{V}\right)=\frac{\sqrt{b}}{2}\left(\gamma(X) \eta\left(Y^{C}\right)\right)_{(x, \omega)}, \\
& \widetilde{F}_{(x, \omega)}\left(\alpha^{V}, Y^{C}, Z^{C}\right)=\frac{\sqrt{b}}{2}\left(\alpha(Z) \eta\left(Y^{C}\right)-\alpha(Y) \eta\left(Z^{C}\right)\right)_{(x, \omega)}, \\
& \widetilde{F}_{(x, \omega)}\left(\alpha^{V}, \beta^{V}, Z^{C}\right)=\widetilde{F}_{(x, \omega)}\left(X^{C}, \beta^{V}, \gamma^{V}\right)=\widetilde{F}_{(x, \omega)}\left(\alpha^{V}, \beta^{V}, \gamma^{V}\right)=0 .
\end{aligned}
$$

Finally, using (4.45) and (4.46), we obtain

$$
\begin{aligned}
& \widetilde{F}_{(x, \omega)}\left(X^{C}+\alpha^{V}, Y^{C}+\beta^{V}, Z^{C}+\gamma^{V}\right) \\
& =\left(\widetilde{F}^{\prime}+\widetilde{F}^{\prime \prime}+\widetilde{F}^{\prime \prime \prime}\right)_{(x, \omega)}\left(X^{C}+\alpha^{V}, Y^{C}+\beta^{V}, Z^{C}+\gamma^{V}\right),
\end{aligned}
$$

where

$$
\begin{gathered}
\widetilde{F}_{(x, \omega)}^{\prime}\left(X^{C}+\alpha^{V}, Y^{C}+\beta^{V}, Z^{C}+\gamma^{V}\right)=\frac{2 a}{\sqrt{b} \omega(\xi)}\{\sqrt{b} \omega(\xi) \omega(R(Z, Y) X) \\
\left.-\omega(R(Z, \xi) X) \eta\left(Y^{C}\right)+\omega(R(Y, \xi) X) \eta\left(Z^{C}\right)\right\}_{(x, \omega)}, \\
\widetilde{F}_{(x, \omega)}^{\prime \prime}\left(X^{C}+\alpha^{V}, Y^{C}+\beta^{V}, Z^{C}+\gamma^{V}\right) \\
=-\frac{\sqrt{b}}{2 a}\left\{-\eta\left(Y^{C}\right)\left[\bar{g}\left(X^{C}, Z^{C}\right)+a \alpha(Z)+a \gamma(X)\right]\right. \\
\left.+\eta\left(Z^{C}\right)\left[\bar{g}\left(X^{C}, Y^{C}\right)+a \alpha(Y)+a \beta(X)\right]\right\}_{(x, \omega)}, \\
\widetilde{F}_{(x, \omega)}^{\prime \prime \prime}\left(X^{C}+\alpha^{V}, Y^{C}+\beta^{V}, Z^{C}+\gamma^{V}\right) \\
=\frac{2 a}{\sqrt{b}(\omega(\xi))^{2}}\left\{-(X f)(Z f) \eta\left(Y^{C}\right)+(X f)(Y f) \eta\left(Z^{C}\right)\right\}_{(x, \omega)} .
\end{gathered}
$$

By direct calculations we verify that for $\widetilde{F}^{\prime}, \widetilde{F}^{\prime \prime}$ and $\widetilde{F}^{\prime \prime \prime}$ the conditions (4.18), (4.19) and (4.24) hold, respectively. 
(i) The assumption that $M$ is flat implies $\widetilde{F}^{\prime}=0$. If $\operatorname{dim} M=2$, then $\operatorname{dim} \widetilde{H}_{t}=3$ and from Proposition 4.2 it follows that $\widetilde{F}^{\prime}$ vanishes too. Hence, $\widetilde{F}=\widetilde{F}^{\prime \prime}+\widetilde{F}^{\prime \prime \prime}$ which means that $\widetilde{H}_{t}$ belongs to the class $\mathbb{G}_{5} \oplus \mathbb{G}_{10}$.

(ii) In the case when $M$ is not flat and $\operatorname{dim} M>2$ we have $\widetilde{F}=\widetilde{F}^{\prime}+\widetilde{F}^{\prime \prime}+\widetilde{F}^{\prime \prime \prime}$. Therefore $\widetilde{H}_{t} \in \mathbb{G}_{4} \oplus \mathbb{G}_{5} \oplus \mathbb{G}_{10}$.

According to the assertion (i) from Theorem 4.1, $\widetilde{H}_{t}$ is paracontact metric in both cases (i) and (ii) if and only if $\mathbb{G}_{5}=\overline{\mathbb{G}}_{5}$. From (4.49) we find $\theta_{\widetilde{F}^{\prime \prime}}(\bar{\xi})=-\frac{(n-1) \sqrt{b}}{a}$. Taking into account the definition of $\overline{\mathbb{G}}_{5}$ in the case when $\phi(X, Y)=g(X, \varphi Y)$, we conclude that $\widetilde{F}^{\prime \prime}$ satisfies the characteristic condition of the class $\overline{\mathbb{G}}_{5}$ if and only if $-\frac{(n-1) \sqrt{b}}{a}=-2(n-1)$. The last equality is equivalent to $b=4 a^{2}$, which completes the proof.

Now, we consider the function $\bar{f}: T^{*} M \longrightarrow \mathbb{R}$ defined in [3] by

$$
\bar{f}=\xi^{V},
$$

or equivalently by $\bar{f}(x, \omega)=\omega_{x}\left(\xi_{x}\right)$ for any $(x, \omega) \in T^{*} M$.

Let

$$
H_{t}=\bar{f}^{-1}(t)=\left\{(x, \omega) \in T^{*} M: \bar{f}(x, \omega)=t, t \in \mathbb{R} \backslash\{0\}\right\}
$$

be the hypersurfaces level set in $T^{*} M$, endowed with the restriction $g$ of the proper natural Riemann extension $\bar{g}$ on $T^{*} M$.

We note that the hypersurfaces level set $H_{t}$ in $T^{*} M$ defined in [3] is a particular case from the set $\widetilde{H}_{t}$ which is obtained by $f=$ const. In [3] it is shown that:

(1) At any point $(x, \omega)$ of $H_{t}$ the gradient of the function $\bar{f}$ is a normal vector field to $H_{t}$ and it is given by

$$
\operatorname{grad} \bar{f}=\frac{1}{a}\left\{\xi^{C}-\frac{b}{a} \xi^{V} W\right\}
$$

(2) The restriction $g$ of $\bar{g}$ on $H_{t}$ is non-degenerate on $H_{t}$, i.e. $\left(H_{t}, g\right)$ is a semiRiemannian hypersurface of $T^{*} M$.

(3) The vertical lift $\alpha^{V}$ of an 1-form $\alpha$ on $M$ and the complete lift $X^{C}$ of $X \in \chi(M)$ are tangent to $H_{t}$ if at any point $(x, \omega) \in H_{t}$ they satisfy the conditions:

$$
\alpha_{x}\left(\xi_{x}\right)=0, \quad \omega_{x}\left(\left(\nabla_{\xi} X\right)_{x}\right)=0 .
$$

We remark that the above three results are immediate consequences from Theorem 4.3 .

From (4.34) we obtain that by $b>0$ the vector field $N$ given by

$$
N=\frac{1}{\sqrt{b} \xi^{V}}\left\{\xi^{C}-\frac{b}{a} \xi^{V} W\right\}
$$

is a time-like unit normal vector field to $H_{t}$. We endow the hypersurface $H_{t}$ of $\left(T^{*} M, P, \bar{g}\right)$ with the almost paracontact metric structure defined by (4.27). By 
using (4.35) we get:

$$
\begin{aligned}
& \bar{\xi}=\frac{1}{\sqrt{b} \xi^{V}} \xi^{C}, \quad \eta\left(X^{C}\right)=\sqrt{b} X^{V}, \quad \eta\left(\alpha^{V}\right)=0 \\
& \varphi X^{C}=X^{C}+2 C(\nabla X)-\frac{X^{V}}{\xi^{V}} \xi^{C}, \quad \varphi \alpha^{V}=-\alpha^{V} .
\end{aligned}
$$

Theorem 4.5. For the $(2 n-1)$-dimensional almost paracontact metric manifold $\left(H_{t}, \varphi, \bar{\xi}, \eta, g\right)$ of $\left(T^{*} M, P, \bar{g}\right)$ with a time-like unit normal vector field $N$ and an almost paracontact metric structure given by (4.52) and (4.53), respectively, we have:

(i) If $M$ is flat or $\operatorname{dim} M=2$, then $H_{t} \in \mathbb{G}_{5}$ and hence $H_{t}$ is quasi-para-Sasakian. In this case $H_{t}$ is para-Sasakian if and only if $b=4 a^{2}$.

(ii) If $M$ is not flat and $\operatorname{dim} M>2$, then $H_{t} \in \mathbb{G}_{4} \oplus \mathbb{G}_{5}$. In this case $H_{t}$ is $K$-paracontact metric if and only if $b=4 a^{2}$.

Proof. We find the tensor field $\widetilde{F}$ of $H_{t}$ by using (4.47), (4.48), (4.49) and (4.50), taking into account that $f=$ const. For arbitrary $X^{C} \in \chi\left(H_{t}\right)$ the equality (4.51) implies $\nabla_{\xi} X=0, X \in \chi(M)$. From the last equality and $\nabla \xi=0$ it follows that $R(Z, \xi) X=R(X, \xi) Z=0, \quad X, \xi, Z \in \chi(M)$. Then the tensor field $\widetilde{F}^{\prime}$, defined by (4.48), becomes

$$
\widetilde{F}_{(x, \omega)}^{\prime}\left(X^{C}+\alpha^{V}, Y^{C}+\beta^{V}, Z^{C}+\gamma^{V}\right)=2 a \omega_{x}\left(R_{x}(Z, Y) X\right) .
$$

One can easily check that $\widetilde{F}^{\prime}$ given by (4.54) satisfies (4.18). Since $f=$ const the tensor field $\widetilde{F}^{\prime \prime \prime}$, defined by (4.50), vanishes. Consequently, for the tensor field $\widetilde{F}$ of $H_{t}$ we have

$$
\begin{aligned}
& \widetilde{F}_{(x, \omega)}\left(X^{C}+\alpha^{V}, Y^{C}+\beta^{V}, Z^{C}+\gamma^{V}\right) \\
& =\left(\widetilde{F}^{\prime}+\widetilde{F}^{\prime \prime}\right)_{(x, \omega)}\left(X^{C}+\alpha^{V}, Y^{C}+\beta^{V}, Z^{C}+\gamma^{V}\right),
\end{aligned}
$$

where $\widetilde{F}^{\prime}$ and $\widetilde{F}^{\prime \prime}$ are determined by (4.54) and (4.49), respectively.

(i) Let us assume that $M$ is flat or $\operatorname{dim} M=2$. Then $\widetilde{F}^{\prime}=0$ and from (4.55) we obtain that $H_{t} \in \mathbb{G}_{5}$. Hence, according to the assertion (iv) from Theorem 4.1, $H_{t}$ is quasi-para-Sasakian. Applying the assertion (ii) from Theorem 4.1 we conclude that $H_{t}$ is para-Sasakian if and only if $\mathbb{G}_{5}=\overline{\mathbb{G}}_{5}$. Analogously as in Theorem 4.4 we establish that it is equivalent to $b=4 a^{2}$.

(ii) In the case when $M$ is not flat and $\operatorname{dim} M>2$ the equality (4.55) holds which means that $H_{t} \in \mathbb{G}_{4} \oplus \mathbb{G}_{5}$. By using the assertion (iii) from Theorem 4.1 we complete the proof.

\section{REFERENCES}

[1] C.-L. Bejan, A classification of the almost Para-Hermitian manifolds, Differential Geometry and its Applications (N. Bokan et al., eds.), Proc. of the Conf. Dubrovnik, 1988, Univ. Novi Sad, Inst. of Mathematics, Novi Sad, 23-27, (1989).

[2] C.-L. Bejan, S. Eken, A characterization of the Riemann extension in terms of harmonicity, Czechoslovak Mathematical Journal 67 (142), 197-206, (2017). 
[3] C.-L. Bejan, S. E. Meric and E. Kilic, Einstein metrics induced by natural Riemann extensions, Adv. Appl. Clifford Algebras , , (2017).

[4] C.-L. Bejan, O. Kowalski, On some differential operators on natural Riemann extensions, Ann. Glob. Anal. Geom. 48, 171-180, (2015).

[5] O. Kowalski, M. Sekizawa, On natural Riemann extensions, Publ. Math. Debrecen 78, 709-721, (2011).

[6] E. M. Patterson, A. G. Walker, Riemannian extensions, Q. J. Math. Oxford Ser. 2 (3), 19-28, (1952).

[7] E. Garcia-Rio, L. Vanhecke, M. E. Vazquez-Abal, Harmonic endomorphism fields, Illinois J. Math. 41, 23-30, (1997).

[8] M. Sekizawa, Natural transformations of affine connections on manifolds to metrics on cotangent bundles, In: Proceedings of 14th Winter School on Abstract Analysis (Srni, 1986), Rend. Circ. Mat. Palermo 14, 129-142, (1987).

[9] T. J. Willmore, An introduction to Differential Geometry, Clarendon Press, Oxford, (1959).

[10] K. Yano, S. Ishihara, Tangent and cotangent bundles, Differential Geometry, Pure and Applied Mathematics 16, Marcel Dekker, New York, (1973).

[11] K. Yano, E. M. Patterson, Vertical and complete lifts from a manifold to its cotangent bundle, J. Math. Soc. Jpn. 19, 91-113, (1967).

[12] S. Zamkovoy, G. Nakova, The decomposition of almost paracontact metric manifolds in eleven classes revisited, J. Geom. (2018) 109:18, https://doi. org / 10.1007/s 00022-018-0423-5

"Gh. Asachi" Technical University of IAsi, Department of Mathematics, 700506 Iasi, ROMANIA

E-mail address: bejanliv@yahoo.com

University of Veliko Tarnovo "St. Cyril and St. Methodius", Faculty of Mathematics and Informatics, Department of Algebra and Geometry, 2 Teodosit Tarnovski Str., Veliko Tarnovo 5003, Bulgaria

E-mail address: gnakova@gmail.com 